\title{
Book Review: \\ Desk Book of Pharmaceutical Dissolution Science and Applications
}

Edited by Sandip B. Tiwari, Umesh V. Banakar, and Vinod Shah

e-mail:vagray@rcn.com

Published by the Society for Pharmaceutical Dissolution Science (SPDS), Mumbai, India

Vivian A. Gray

Dissolution Technologies

Hockessin, DE 19707

A $s$ the editors indicate in the forward, this book is intended to address the state of dissolution testing beyond tablets and capsules dosage forms. The book, supported by the Society for Pharmaceutical Dissolution Science (SPDS), covers the current status of dissolution science related to such timely topics as bioavailability, mathematical treatment of data, novel dosage forms, international guidelines and harmonization, automation, and other important dissolution topics. The book's 265 pages comprise 15 chapters; each chapter has a unique perspective and updated material that will be of great utility for the dissolution analyst. The authors of the chapters are well chosen and can provide not only concise descriptions of the topics but also offer insight into emerging trends.

The first chapter, "Historical Highlights and the need for Dissolution Testing" by Vinod Shah, begins with the history of the test and quickly moves to the resurgence of interest in improving the predictive capability and understanding the science of dissolution testing. He reiterates that the test is well established, reliable, and reproducible and is increasingly relied upon to assure product performance. Shah makes an important point that the test is simple, economical, and a great utility in developing countries to assure acceptable drug product quality.

The next chapter, "Dissolution Science: Theories and Applications" by Umesh Banakar, discusses the science behind dissolution by providing mathematical models and theories that assist in understanding the mechanism of dissolution of the active ingredient from the solid dosage form. Banakar emphasizes the dissolution performance data must be adequate to characterize the rate and extent of the drug released over time for complete performance. The results should be reproducible and able to be validated. This discussion continues into Chapter 3, titled "Dissolution-Methodology and Scientific Principles" and written by Suresh Venkataram covering the physicochemical principles of the drug substance and providing a detailed analysis of factors that affect dissolution from the perspective of product related considerations. Venkataram examines the evolution of the apparatus, namely the addition of Apparatus 3 and 4 and also explores the hydrodynamic issues involved in the apparatus.

Chapters 4, 5, and 6 all delve into the realm of bioavailability, bioequivalence (BE), and in vivo and in vitro relationships. Chapter 4, "Dissolution and Bioavailability: In Vitro-In Vivo Correlations (IVIVC), Bioavailability, and Bioequivalence" by Banakar, focuses on IVIVC. He describes the models behind IVIVC and explains the biopharmaceutics classification system (BCS). There is an in-depth discussion of the relevant physicochemical and physiological parameters that influence drug dissolution in the gastrointestinal tract. Banakar describes the techniques available for evaluating in vivo dissolution rates and defines the various techniques for correlating dissolution and bioavailability. He poses the fundamental question of whether it is possible to simulate in vivo conditions within the in vitro dissolution test in the laboratory and admits that it is surely challenging. Chapter 5, "In Vitro-In Vivo" Correlation: Applications and Limitations" by Jean-Michel Cardot, gives practical information on definitions, including the various levels (Level A, B, C, and D) and limits of IVIVC. Cardot discusses the requirements and assumptions of IVIVC, and he states that to establish and validate correlations, more than one formulation needs to be used and that at least three formulations are necessary on Level A. His figure depicting internal and external predictability is especially useful. The chapter also presents a discussion of the role of simulations. He explores the use of IVIVC for generic products. Chapter 6, "Comparison of Nonclinical Permeability Techniques for BCS ClassificationTools to Augment Dissolution Outcomes" by Vatsala Naageshwaran and Sid Bhoopathy, offers an in-depth 
discussion of the impetus for biowaivers and explores the notion that, contrary to popular belief, the traditional approach of BE studies may not always be the easiest or most successful path toward developing interchangeable immediate-release products. The authors state this is because each molecule has its own unique challenges, and a human pharmacokinetic study may not always be the right option from an ethical, cost, or scientific perspective. Naageshwaran and Bhoopathy explain there is global acceptance of the BCS biowaiver system and compare regulatory agencies and their specifications for a BCS-based biowaiver. They also devote a good part of the chapter to the models for the investigation and prediction of drug absorption.

Readers of Dissolution Technologies who work at the bench will likely find Chapters 7 and 8 especially interesting. Chapter 7, "Automation of Dissolution Testing" by Samir Haddouchi, gives an excellent in-depth analysis of automation. Haddouchi discusses the various levels of automation and notes that, whereas all pharmacopeias allow for the implementation of automation, they still require the end user to demonstrate equivalence to manual methods. This chapter provides a comprehensive list of the aspects of an entirely automated system. The topics of automated method transfer, cross-validation, and cleaning validation are especially useful and not usually covered in guidance documents. The use of automation can be of great help to improve efficiency with the lab, and this chapter is a welcome practical addition to the book. Chapter 8, "A Review of Dissolution Testing with Flow-Through Cell (USP Apparatus 4)" by Jean-Marie Glantzmann and Jean-Louis Raton, is a useful treatment of Apparatus 4 for the dissolution scientist who may not be familiar with the instrument. This chapter features a detailed description of the operation and utility of Apparatus 4, including figures and tables depicting the many specialized cell types for different dosage forms. One table (Table 3 ) presents a very useful listing of the validation parameters, including testing intervals. The authors also explore the latest application developments such as apparent dissolution, a flowthrough cell for lipophilic dosage forms, a flow-through cell for microspheres, and drug-eluting stents.

Chapter 9, "Compendial Requirements for Dissolution: A Look at USP/EP and JP Pharmacopeias" by Hakan Eroglu, Erem Bilensoy, and A. Atilla Hincal is a comprehensive analysis of the similarities and differences of the three pharmacopeias. The authors provide a remarkable comparison of the dissolution procedure and requirements in each pharmacopeia in Table 1, which will be of great assistance to those international companies who provide drug products on a global scale. Another table in this chapter, Table 7, compares the major variances among the three compendia regarding the interpretation of dissolution. These two tables alone are worth the purchase of this book. The authors end the chapter stating the expectation that academia and industry will continue to work with regulatory bodies in enhancing and harmonizing these dissolution requirements globally. We hope he is correct.

The next two chapters deal with dissolution testing of two specialized dosage forms. In Chapter 10, "In Vitro Dissolution Testing of Nanoparticulate Drug Delivery Systems," Mangal Nagarsenker, Vivek Dhawan, and Sanket Shah provide a list of important considerations in drug release testing methodology for nanoparticles, a unique and fairly new dosage form. The chapter is full of numerous practical examples, including methods where there are special modifications to the dissolution test to accommodate the challenges posed by nanoparticulates. The authors note the lack of a standardized compendial method, which has led to a variety of modifications, and hope for the development of a standard apparatus or uniform modification of a compendial apparatus such as the flow-through cell in the near future. Chapter 11, "Drug Release from Semisolid Dosage Forms" by Flavian Stefan Radulescu and Dalia Simona Miron, discusses the methods and apparatus that are used for testing semisolid dosage forms. In the relatively new USP General Chapter $<1724>$ Semisolid Dosage Forms-Performance Tests, the following apparatus are described: vertical diffusion cell, immersion cells, and flow-through cell adaptations. The critical selection of membrane and receptor composition is explored in this chapter along with the testing parameters. There is also an FDA guidance, SUPAC-SS, that provides in-depth information on the evaluation of changes for topical semisolid dosage forms using the in vitro release (IVR) test. The authors explore similarity assessments unique to IVR and note the difficulty of accomplishing IVIVC with this dosage form.

The next two chapters deal with the mathematical treatment of dissolution curves. Chapter 12, "Comparative Analysis of Dissolution Data" by Constantin Mircioiu, discusses the critical tools of dissolution profile comparison. The chapter has several useful figures that give instructive examples. Figure 1 gives examples of two different mean curves whose difference is either statistically significant or non-significant. Figure 2 illustrates the variability of points and, in particular, the impact of variability at the 5 -min time point. Figure 3 
illustrates two clusters of curves corresponding to a reference and tested drug with a difference due to the variability of the distribution of curves in space. Figure 5 shows the aggregation of curves in a different cluster due to differences in the dissolution of the drug in vivo. Figure 10 illustrates possible outlier values in the evaluation of single point versus entire dissolution curves. Figures 11 , 12 , and 13 are devoted to IVIVC. The chapter gives a nod to the analyst by stating that the first step in comparison is the "gold standard" of visual inspection. The second step is the application of mathematical methods with the third step of phenomenological validation. Chapter 13, "Mathematical Treatment of Dissolution Data for Extraction of Vital Product Information" by Mukesh Gohel, Sandip Tiwari, and Jayvadan Patel, gives special emphasis to the study of the active pharmaceutical ingredient. The authors explain several models that deal with the kinetics of drug release including the zero-order model, first-order model, Higuchi model, KorsmeyerPeppas model, Hixson-Crowell model, quadratic model, and Weibull model. They mention alternatives such as the DDsolver, artificial neural networks, and partial least squares regression. The chapter also provides several practical examples of mathematical model fitting and its application to drug release data. The chapter closes with the sentiment that mathematical treatment of dissolution data can be beneficial to the formulators, industry, and regulators, and the application of statistics to dissolution data can also aid in rationalizing formulation performance and selecting the most appropriate formulation for bioequivalence studies or clinical trials.

Chapter 14, "Dissolution Testing of Generic Products: Regulatory Perspective" by H. V. Raghunandan, J. Harsha, and B. K. Mahesh, takes a global view of the dissolution data requirements. Table 1 provides a good summary of the FDA requirements for dissolution requirements for an NDA and ANDA. The authors also describe the guidelines for bioequivalence from the European Medicines Agency, Japanese Pharmacopeia, Australian Therapeutics Goods Administration, Health Canada, South African Medicines Control Council, New Zealand Medicines \& Medical Devices Safety Authority Guidance, World
Health Organization guidance, and the Indian Regulatory Guidance. The conclusions state that regulatory agencies, as a general practice, emphasize a scientific and rational justification for the choice of dissolution media, test conditions, and subsequent biowaivers.

Vijay Kshirsagar, the author of the final chapter titled "Investigation of Out-of-Specification (OOS) Results Obtained in Dissolution Testing," brings nearly 40 years of experience with various national and international pharmaceutical companies to the book, and he draws on his experience interacting with inspectors for all the major entities of the global market. Table 1 is a useful listing of the top 10 deficiency categories according to the Medicines and Health Products Regulatory Agency of the United Kingdom. Kshirsagar relates an interesting narrative on the "Barr Decision," a landmark U.S. court case. He also provides a comprehensive list of factors that should be examined during a dissolution OOS investigation, which includes deaeration, calibration, filter validation, method interference, and changes in the approved product. Figure 1 provides a fault-tree approach for investigations of OOS dissolution, and Figure 2 offers a flow chart for OOS dissolution for drug products. The information in Figures 1 and 2 are reason enough for purchasing this book. Also, Table 6 lists probable root causes for stability failures in dissolution testing-information that is quite interesting and helpful.

Overall, the book packs a wealth of information in only 265 pages. It has something for everyone, from the formulator who is interested in IVIVC, to the lab analyst needing guidance in method development, to the manager needing help with an OOS. I highly recommend this book to practitioners of dissolution testing and anyone with a special interest in dissolution testing, as their needs will be served by several of the more specialized chapters.

To purchase this book, please contact Rahul Gavankar of United Books and Periodicals at gavankar@ubp.co.in and unitedbooks@gmail.com. 